\title{
Faster simulated laparoscopic cholecystectomy with haptic feedback technology
}

This article was published in the following Dove Press journal:

Open Access Surgery

3 October 2011

Number of times this article has been viewed

\section{Marina Yiasemidou \\ Daniel Glassman \\ Peter Vasas \\ Sarit Badiani \\ Bijendra Patel}

Barts and the London School of Medicine and Dentistry, Department of Upper GI

Surgery, Barts and The Royal

London Hospital, London, UK
Correspondence: Marina Yiasemidou I0 Blackwell Gardens, Edgware, Middlesex, HA8 8QA, UK Tel +44 $797553 \quad 1067$

Email marinayiasemidou@gmail.com
Background: Virtual reality simulators have been gradually introduced into surgical training. One of the enhanced features of the latest virtual simulators is haptic feedback. The usefulness of haptic feedback technology has been a matter of controversy in recent years. Previous studies have assessed the importance of haptic feedback in executing parts of a procedure or basic tasks, such as tissue grasping. The aim of this study was to assess the role of haptic feedback within a structured educational environment, based on the performance of junior surgical trainees after undergoing substantial simulation training.

Methods: Novices, whose performance was assessed after several repetitions of a task, were recruited for this study. The performance of senior house officers at the last stage of a validated laparoscopic cholecystectomy curriculum was assessed. Nine senior house officers completed a validated laparoscopic cholecystectomy curriculum on a haptic simulator and nine on a nonhaptic simulator. Performance in terms of mean total time, mean total number of movements, and mean total path length at the last level of the validated curriculum (full procedure of laparoscopic cholecystectomy) was compared between the two groups.

Results: Haptic feedback significantly reduced the time required to complete the full procedure of laparoscopic cholecystectomy (mean total time for nonhaptic machine 608.83 seconds, mean total time for haptic machine 553.27 seconds; $P=0.019$ ) while maintaining safety standards similar to those of the nonhaptic machine (mean total number of movements: nonhaptic machine 583.74, haptic machine $603.93, P=0.145$, mean total path length: for nonhaptic machine 1207.37 $\mathrm{cm}$, for haptic machine $1262.36 \mathrm{~cm}, P=0.101)$.

Conclusion: Haptic feedback significantly reduced the time required to complete the full procedure of laparoscopic cholecystectomy.

Keywords: simulation training, surgical skills, haptic feedback, surgical training

\section{Introduction}

Virtual reality simulators have been gradually introduced into surgical training. Their role has been enhanced by enforcement of the European working time directive and increasing emphasis on patient safety. One of the features of the latest virtual simulators is haptic feedback. Tactile response, as it is otherwise known, imitates the forces a surgeon experiences in theater. Usefulness of haptic feedback technology has been a matter of controversy for several years. Study outcomes have been contradictory. ${ }^{1-10}$ Previous projects have focused on assessing the lifelikeness or importance of haptic feedback in executing parts of a procedure or basic tasks, such as tissue grasping or cutting. ${ }^{1-9}$ Most of the studies conducted have used novices, specifically medical students, who had not had any substantial simulation 
training or previous theater experience. Their performance was assessed after a random number of repetitions of the same task. ${ }^{1-3,9}$ Being evaluated at the beginning of their simulation training avoids the "learning curve effect", and having no theater experience contributes further to lack of progression in acquiring laparoscopic skills. For this study, we recruited junior trainees at the senior house officer level, who were simulation training novices. Their performance was assessed at the very last stage of a three-level, validated laparoscopic cholecystectomy curriculum. The aim of the study was to assess the role of haptic feedback within a structured educational environment, based on the performance of junior surgical trainees after undergoing substantial simulation training, within a curriculum proven to follow a "learning curve". 11

\section{Materials and methods}

The curriculum used for this study is described by Darzi et $\mathrm{al}^{11}$ and consists of three levels, ie, a basic task level, a procedural task level, and the full procedure of laparoscopic cholecystectomy. The first level includes nine basic tasks (Table 1), the second level comprises four procedural tasks (Table 2), and the third and final level includes the full procedure of laparoscopic cholecystectomy. In order for the trainee to progress towards completion of the entire curriculum, they should fulfill the requirements for completion of each level separately.

\section{Level I}

For level 1 , initially all nine basic tasks are performed twice in two sessions more than 1 hour apart on the same day. Two of the basic tasks (clipping and grasping and two-hand maneuvers) are then performed in the same timely manner as before. The following metrics should be achieved on two consecutive sessions in order for the trainee to progress to the next level of the curriculum:
- Clipping and grasping, with total time taken $<100$ seconds

- Two-handed maneuvers, with total time taken $<90$ seconds, total number of movements $<100$, and total path length $<440 \mathrm{~cm}$

\section{Level 2}

In level 2, four procedural tasks are performed twice in two sessions more than 1 hour apart on the same day. Two of the procedural tasks (Calot's triangle dissection and gallbladder separation) are then performed twice in two sessions more than 1 hour apart on the same day. The following metrics should be achieved on two consecutive sessions in order for the trainee to progress to the next level:

- Calot's triangle dissection, with total time taken $<280$ seconds, total number of movements $<240$, and total cautery time $<15$ seconds

- Gall bladder separation, with total time taken $<300$ seconds, total number of movements $<275$, and total path length $<500 \mathrm{~cm}$

\section{Level 3}

In level 3, the full procedure of laparoscopic cholecystectomy is performed in two different sessions more than 1 hour apart on the same day. For this level and the whole curriculum to be completed and proficiency attained, the following metric should be achieved in two consecutive sessions:

- Full laparoscopic cholecystectomy, with total time taken $<540$ seconds, total number of movements $<480$, and total path length $<1000 \mathrm{~cm}^{11}$

\section{Virtual reality simulators}

The virtual reality surgical simulators used for this study belong to the LAP Mentor ${ }^{\mathrm{TM}}$ family (Simbionix Corporation, Cleveland, $\mathrm{OH}$ ). The LAP Mentor virtual reality laparoscopic surgical simulator is enhanced with haptic feedback technology. LAP Mentor Express is a practical

Table I Description of basic tasks

\begin{tabular}{ll}
\hline Task & Description \\
\hline Camera manipulation $0^{\circ}$ & Using $0^{\circ}$ find and photograph a number of red balls in various points of the screen \\
Camera manipulation $30^{\circ}$ & Using $30^{\circ}$ find and photograph a number of red balls in various points of the screen \\
Eye-hand coordination & Touch flashing balls of the red and blue color with the diathermy hook of the same color \\
Clip applying & Clip leaking ducts within a green colored stripe. One hand can be used in this task \\
Clipping and grasping & Grasp and extend leaking duct with grasper and clip \\
Two-handed maneuver & Using two graspers expose balls within a gel and transfer them in a metal bowl \\
Cutting & Using grasper and endoscissors detach gel from metal ring \\
Electrocautery & Use hook to cauterize highlighted strings, use accessory hook to retract nonhighlighted bands \\
Object translocation & Place an object in the exact same position as its shadow
\end{tabular}


Table 2 Description of procedural tasks

\begin{tabular}{|c|c|}
\hline Task & Description \\
\hline Clipping and cutting retracted gallbladder & $\begin{array}{l}\text { The gallbladder is exposed with the Hartmann's pouch retracted laterally. Clip } \\
\text { cystic duct and artery. Use the endoscissors to cut the cystic duct and artery }\end{array}$ \\
\hline Clipping and cutting, two hands & $\begin{array}{l}\text { The gallbladder is already exposed. Grasp and pull Hartmann's pouch. Clip } \\
\text { cystic duct and artery. Use endoscissors to cut cystic duct and artery }\end{array}$ \\
\hline $\begin{array}{l}\text { Dissection } \\
\text { (of cystic duct and artery) - achieving a critical view }\end{array}$ & $\begin{array}{l}\text { Grasp gallbladder's infundibulum with left hand and retract away from the } \\
\text { liver, to assure a safe dissection. Dissect the peritoneum covering Calot's } \\
\text { triangle and expose a colored cystic duct and artery }\end{array}$ \\
\hline Gallbladder separation (from liver bed) & $\begin{array}{l}\text { Retract gall bladder from the liver bed. Dissect peritoneal adhesions between } \\
\text { the gallbladder and the liver. Before finishing the dissection, inspect liver bed } \\
\text { for possible bleeding sites and bile leakage }\end{array}$ \\
\hline
\end{tabular}

and portable laparoscopic virtual reality simulator without haptic feedback. The curriculum used for this study was developed on LAP Mentor and, therefore, both simulators incorporated all basic and procedural tasks as well as the full procedure included in the specific curriculum. ${ }^{12}$ There have been several validation studies for the two simulators. Sung et al compared basic laparoscopic skills before and after using the LAP Mentor. They concluded that training on the LAP Mentor is a useful educational process that may lead to improved theater performance. ${ }^{13}$ Reznick et al demonstrated that full procedure training on the LAP Mentor improved surgical skills. Assessment of skills was done by a blinded examiner using a validated assessment scale, ie, the Global Operative Assessment of Laparoscopic Skills. ${ }^{14}$

\section{Study design}

The participants were MSc Surgical Skills and Sciences students from Barts and the London School of Medicine and Dentistry, Queen Mary College, University of London. The inclusion criteria were being a senior surgical house officer, having seen or assisted in less than ten laparoscopic cholecystectomies, and having fulfilled the validated laparoscopic cholecystectomy simulation training curriculum. Students who had already performed a laparoscopic cholecystectomy in theater or had previous experience in simulation training were excluded from the study. Overall, 18 students were recruited (eleven males, seven females). Nine of them fulfilled the validated curriculum described earlier on the nonhaptic simulator and the other nine on the haptic one. The performance of the two nine-member teams was compared during the third and last part of the curriculum. The minimum number of attempts used by a trainee to fulfill the last part of the curriculum was two, therefore the performances of all other trainees were assessed after two attempts. The metrics used to assess performance were the mean total time taken to complete the full procedure, mean total (right hand and left hand) path length of the instrument, and mean total (right hand and left hand) number of instrument movements. Path length is the feature provided by the simulator for the length $(\mathrm{cm})$ that the instruments have moved during performance of a task or procedure.

\section{Data collection}

The aforementioned metrics were measured immediately by the virtual reality simulators and stored under different file names for each team on each simulator. Data for the three metrics were collected from storing software and assessed using SPSS software (v 18; SPSS Inc, Chicago, IL). For each metric assessed, the mean value was calculated for two attempts at laparoscopic cholecystectomy by each trainee. The normality of the distribution of each metric for each team was evaluated using the Shapiro-Wilk test. If distributions were normal, a parametric test was used; if they were not, a nonparametric test was used. The parametric method used was the independent $t$-test and the nonparametric method was the Mann-Whitney test. A $P$ value of $<0.05$ was considered to be statistically significant.

\section{Results}

Eighteen trainees fulfilled the validated laparoscopic cholecystectomy curriculum. Nine did so on a haptic laparoscopic surgical simulator and nine on a nonhaptic simulator. The performance of the two teams was compared during the third part of the curriculum (full laparoscopic cholecystectomy). Metrics used for comparison were mean total time taken to complete the full procedure, mean total path length of instrumental movements, and mean total number of movements (Figures 1, 2, and 3).

The Shapiro-Wilk test used to assess the normality of the distributions showed that the distributions of mean total time for the nonhaptic machine, mean total number of movements for the nonhaptic machine, mean total path length for 


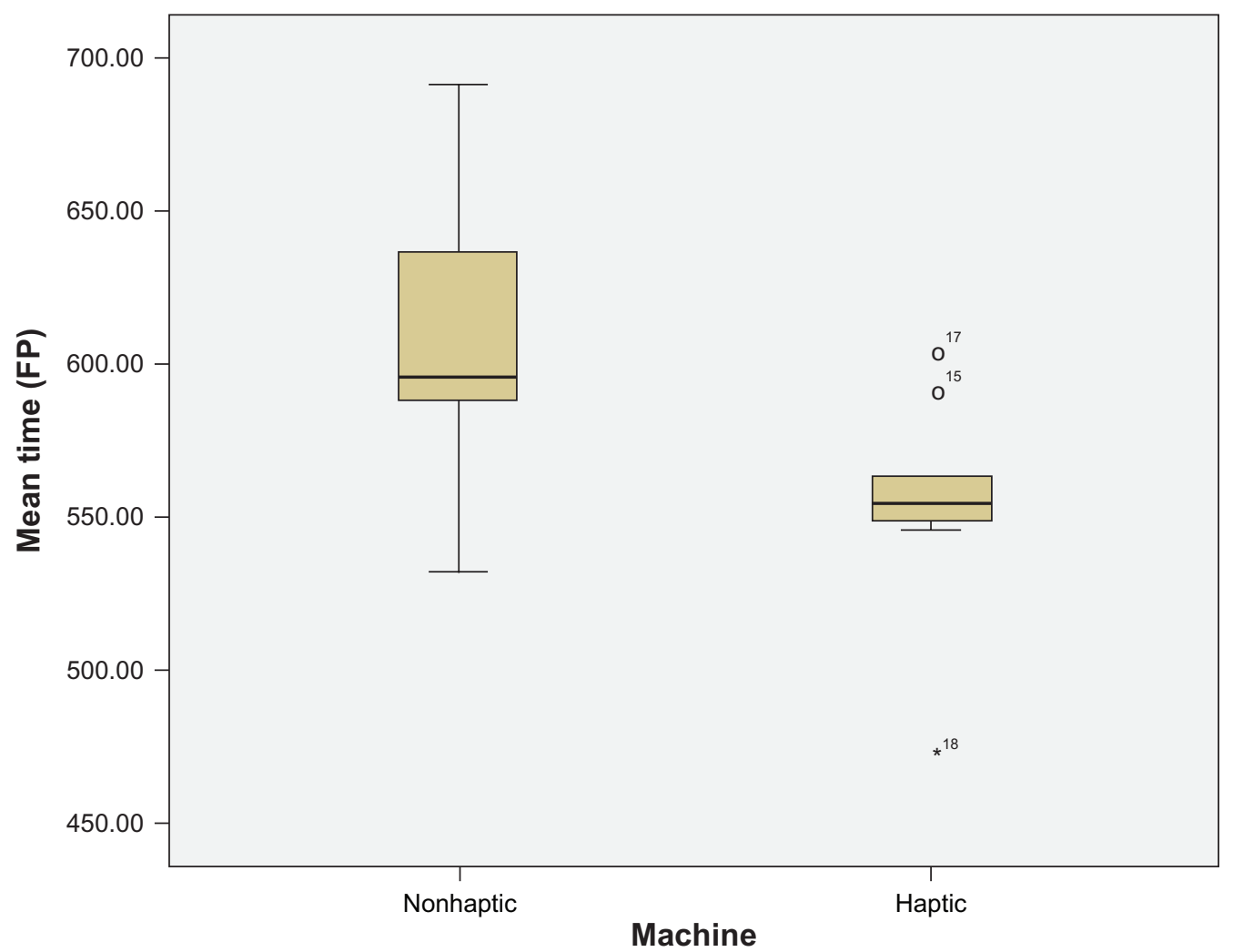

Figure I Distribution of mean total time for full laparoscopic cholecystectomy. Horizontal lines within boxes, boxes and whiskers represent median, interquartile range and range, respectively. Circles and stars represent outliers.

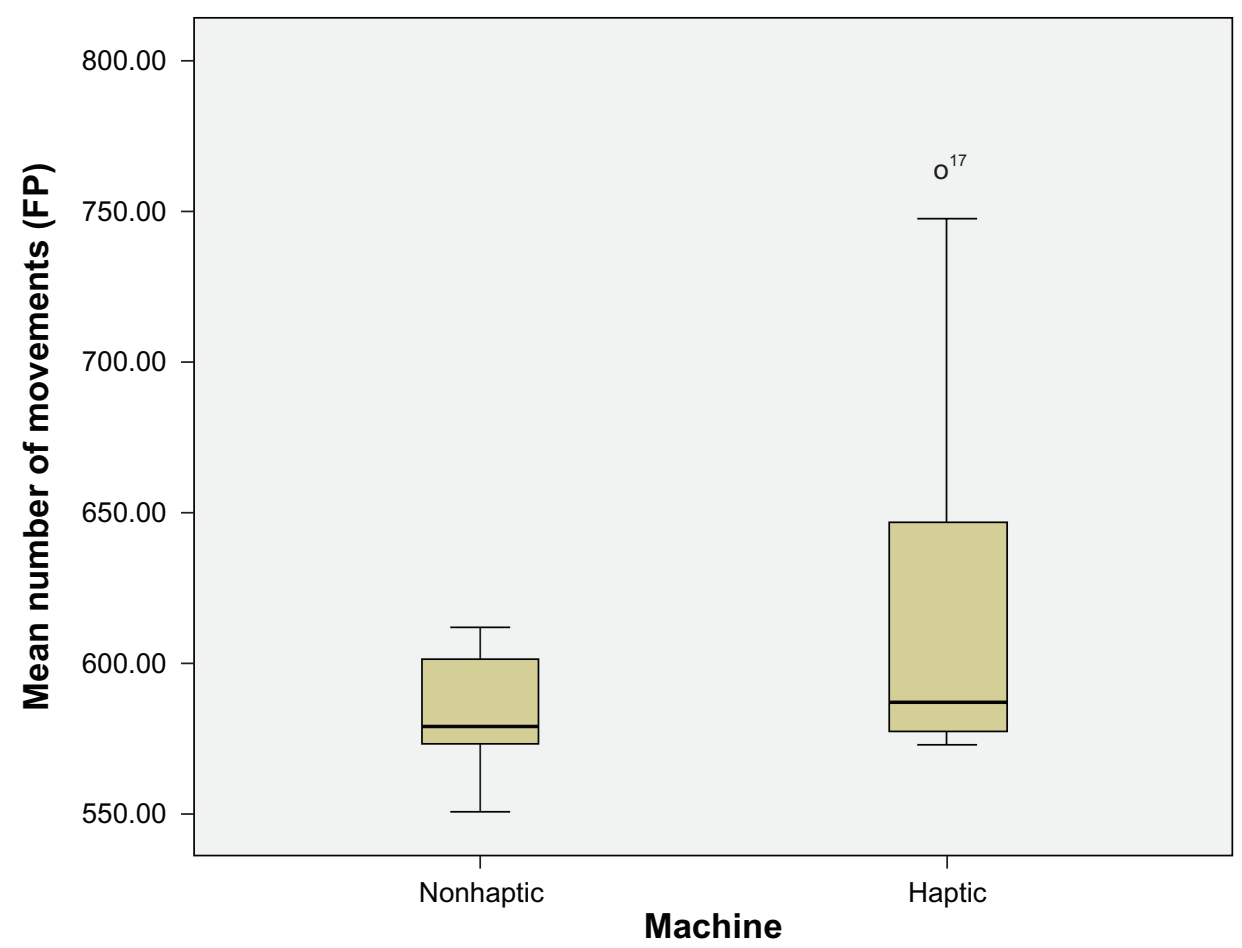

Figure 2 Distribution of mean total number of movements for full procedure (laparoscopic cholecystectomy). Horizontal lines within boxes, boxes and whiskers represent median, interquartile range and range respectively. Circles and stars represent outliers. 


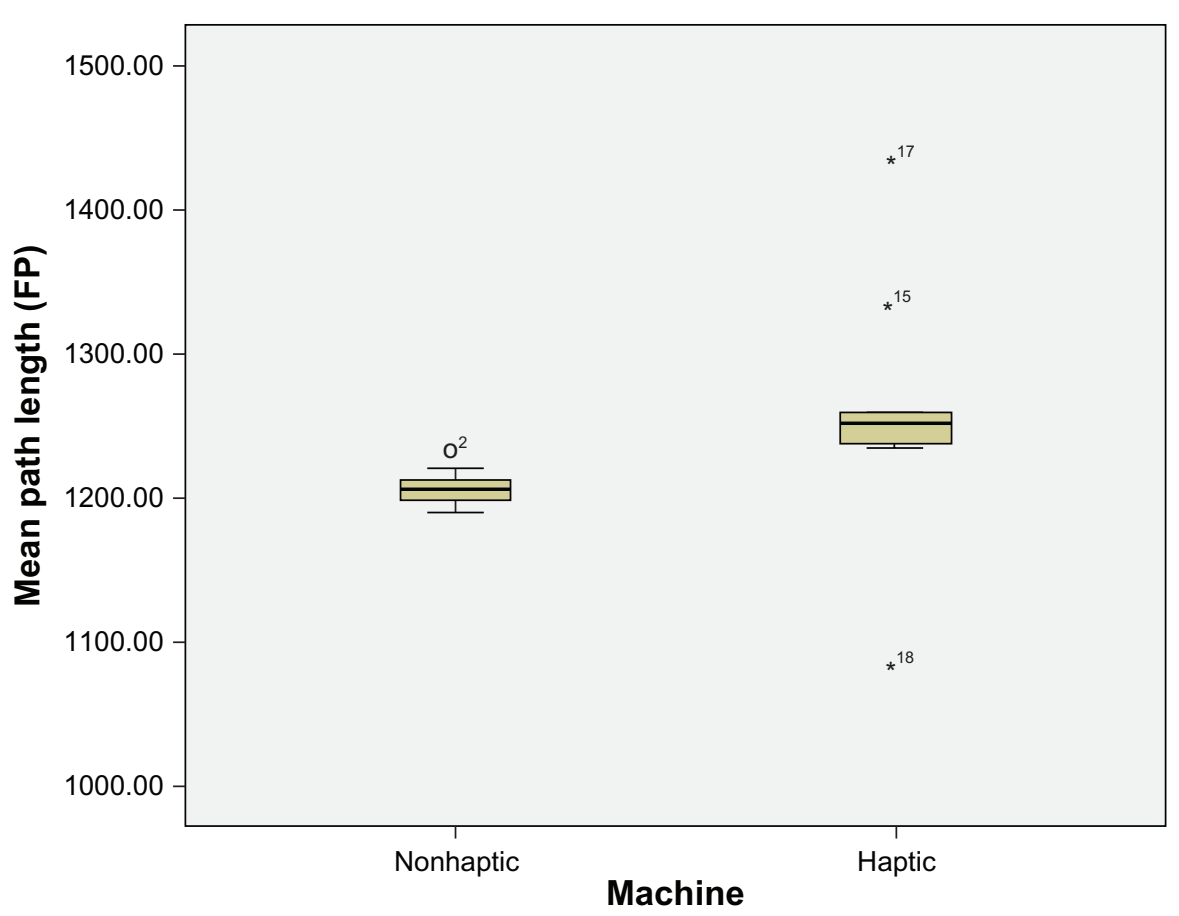

Figure 3 Distribution of mean total path length for full procedure (laparoscopic cholecystectomy). Horizontal lines within boxes, boxes and whiskers represent median, interquartile range and range respectively. Circles and stars represent outliers.

the nonhaptic machine, and mean total path length for the haptic machine were normal, while the remainder were not (Table 3). According to these results, the independent sample $t$-test was used to compare the mean total path length and the Mann-Whitney test was used for the mean total time and mean total number of movements. The Mann-Whitney test results for mean total time showed that the amount of time required to complete the full procedure of laparoscopic cholecystectomy was significantly less on the haptic simulator compared with the nonhaptic simulator (mean total time for nonhaptic machine 608.83 seconds, mean total time for haptic machine 553.27 seconds, $P=0.019$ ). The same test showed that there was no statistically significant difference between the mean total number of movements achieved after training on the nonhaptic machine compared with those achieved after training on the haptic machine (mean for nonhaptic machine 583.74, mean for haptic machine 630.93, $P=0.145$ ). The independent samples $t$-test comparing mean total path

Table 3 Shapiro-Wilk test result

\begin{tabular}{lll}
\hline & Machine & P value \\
\hline Mean total time & Nonhaptic & 0.892 \\
Mean total number of movements & Haptic & 0.048 \\
& Nonhaptic & 0.738 \\
Mean total path length & Haptic & 0.009 \\
& Nonhaptic & 0.775 \\
& Haptic & 0.134 \\
\hline
\end{tabular}

length between the haptic and nonhaptic simulators showed that there was no statistically significant difference in mean total path length achieved after training on the nonhaptic machine comparing with that on the haptic machine (mean for nonhaptic machine $1207.37 \mathrm{~cm}$, mean for haptic machine $1262.36 \mathrm{~cm}, P=0.101)$.

\section{Discussion}

This study aimed to evaluate the role of haptic feedback under structured training conditions. Because simulation training usually involves novices who have some clinical experience, the authors of this study chose to include senior house officer level surgical trainees. Assessing performance within a curriculum shown to follow a learning curve allows for more objective evaluation of the role of haptic feedback in training of novices. Familiarity with haptic technology is given time to develop by evaluating performance at the final level of the curriculum, after successful completion of all previous levels,

The current study showed that haptic feedback significantly reduced the time required to complete the full procedure of laparoscopic cholecystectomy, after substantial training on basic and procedural tasks. The lack of a significant difference between the mean total path length and the mean total number of movements demonstrates that laparoscopic cholecystectomy can be performed faster on the 
haptic simulator and as safely as on the nonhaptic simulator, given that reduced path length and number of movements have been associated with low complication rates.

These results show that sufficient training time should be allowed for haptic feedback impact to take effect. Furthermore, they demonstrate that there is constant progression within a training curriculum which is more rapid and substantial if haptic feedback technology is used. The learning curve seems to be in effect not only within performing the same task but also between different tasks as well. Overall, haptic feedback after a certain "incubation period" can greatly enhance acquired laparoscopic skills, whilst maintaining good safety standards.

\section{Disclosure}

The authors report no conflicts of interest in this work.

\section{References}

1. Hiemstra E, Terveer EM, Chmarra MK, Dankelman J, Jansen FW. Virtual reality in laparoscopic skills training: is haptic feedback replaceable? Minim Invasive Ther Allied Technol. 2011;20(3):179-184.

2. Salkini MW, Doarn CR, Kiehl N, Broderick TJ, Donovan JF, Gaitonde $\mathrm{K}$. The role of haptic feedback in laparoscopic training using the LapMentor II. J Endourol. 2010;24(1):99-102.

3. Panait L, Akkary E, Bell RL, Roberts KE, Dudrick SJ, Duffy AJ. The role of haptic feedback in laparoscopic simulation training. J Surg Res. 2009;156(2):312-316.

4. Choi C, Kim J, Han H, Ahn B, Kim J. Graphic and haptic modelling of the oesophagus for VR-based medical simulation. Int J Med Robot. 2009;5(3):257-266.
5. Westebring-van der Putten EP, Lysen WW, Henssen VD, et al. Tactile feedback exceeds visual feedback to display tissue slippage in a laparoscopic grasper. Stud Health Technol Inform. 2009;142:420-425.

6. Okrainec A, Farcas M, Henao O, et al. Development of a virtual reality haptic Veress needle insertion simulator for surgical skills training. Stud Health Technol Inform. 2009;142:233-238.

7. Lee DH, Choi J, Park JW, et al. An implementation of sensor-based force feedback in a compact laparoscopic surgery robot. ASAIO J. 2009;55(1):83-85.

8. Westebring-van der Putten EP, van den Dobbelsteen JJ, Goossens RH, Jakimowicz JJ, Dankelman J. Effect of laparoscopic grasper force transmission ratio on grasp control. Surg Endosc. 2009;23(4):818-824.

9. Shamsunder SC, Manivannan M. Haptic guided laparoscopy simulation improves learning curve. Stud Health Technol Inform. 2008; 132:454-456.

10. Hagen ME, Meehan JJ, Inan I, Morel P. Visual clues act as a substitute for haptic feedback in robotic surgery. Surg Endosc. 2008; 22(6):1505-1508.

11. Aggarwal R, Crochet P, Dias A, Misra A, Ziprin P, Darzi A. Development of a virtual reality training curriculum for laparoscopic cholecystectomy. Br J Surg. 2009;96(9):1086-1093.

12. Simbionix. LAP Mentor. Available from: http://www.simbionix.com/ LAP_Mentor.html. Accessed September 6, 2011.

13. Kim TH, Ha JM, Cho JW, You YC, Sung GT. Assessment of the laparoscopic training validity of a virtual reality simulator (LAP Mentor ${ }^{\mathrm{TM}}$ ). Korean J Urol. 2009;50(10):989-995.

14. Okrainec A, Tekian A, Aarts M-A, Grantcharov T, Escallon J, Reznick R. Virtual reality training on basic laparoscopic tasks vs virtual reality training of an entire surgical procedure: A randomized controlled trial using real world operations as an outcome. Presented at the Annual Meeting of the Association of Surgical Educators, Salt Lake City, UT, April 28-30, 2009.
Open Access Surgery

\section{Publish your work in this journal}

Open Access Surgery is an international, peer-reviewed, open access journal that focuses on all aspects of surgical procedures and interventions. Patient care around the peri-operative period and patient outcomes post surgery are key topics. All grades of surgery from minor cosmetic interventions to major surgical procedures are covered. Novel techniques Submit your manuscript here: http://www.dovepress.com/open-access-surgery-journal

\section{Dovepress}

and the utilization of new instruments and materials, including implants and prostheses that optimize outcomes constitute major areas of interest. The manuscript management system is completely online and includes a very quick and fair peer-review system. Visit http://www.dovepress.com/ testimonials.php to read real quotes from published authors. 